\title{
An Embedded English Synthesis Approach Based on Speech Concatenation and Smoothing
}

\author{
Gui-Lin Chen Dong-Jian Yue Yi-Qing Zu Zhen-Li Yu \\ China Research Center, Shanghai, Motorola Labs \\ gui-lin.chen@motorola.com \{a17498, a16534, a16300\}@email.mot.com
}

\begin{abstract}
An embedded English synthesis approach based on speech concatenation and smoothing is described. This approach adopts phonetic sub-words as carrier of variable-length units. We define 5-class units to cover all English phonetic phenomena. The corresponding cost function and search procedure based on dynamic programming are addressed in the unit-selection stage. Vocal tract response, pitch value and phase are interpolated and merged at concatenating points for smoothing speech in the synthesis stage. The preliminary test shows that this approach can reach a good balance of naturalness, intelligibility and data footprint.
\end{abstract}

\section{INTRODUCTION}

Embedded text-to-speech(TTS) synthesis as demands in handheld applications increase in recent years. It has received considerable attention[1] [5]. Considering that formant-based speech synthesis is computationally expensive and poor in naturalness, we designed an embedded English synthesis approach based on speech concatenation and smoothing. Due to the limitations from memory size and computational power, we focused on in-word prosody generation and shallow prosodic phrasing in this approach rather than complete prosodic prediction at the sentence level in a largefootprint TTS system. In section 2, a variable-length speech unit is described. In section 3, a search procedure based on cost function and dynamic programming is presented. In section 4, a synthesis approach combining speech concatenation and smoothing is discussed. In section 5, a preliminary test is introduced. Finally, we discussed some existing problems.

\section{UNIT DEFINITION}

It is well known that unit definition influences the synthesis strategy directly. Several following phases of TTS development such as design of speech inventory, unit selection even speech synthesis are closely linked to the unit definition. Typical units used in concatenative systems include di-phone, tri-phone, syllable and variable-length unit[1][2][4]. We employed variable-length units to achieve a balance of naturalness and data footprint. The carrier of the defined unit is phonetic sub-word. In our experience, carrierword-based unit has some noticeable shortcomings. For instance, some phonemes, mainly consonants are assimilated due to rapid transition; some units lack substitutionality due to significant contextual variance and some units lack separability due to severe co-articulation. We call these units as "bad units", which incur unstability and inconsistency in synthetic waveform. However, we can hardly gain the obvious evidence of correspondence between speech quality and spectral parameters in a straightforward way. It is quite challenging in picking up bad units from an inventory automatically. Different from the carrier-word approach, we checked all the phonetic sub-words in the final inventory manually. We deleted some inappropriate units and organized the inventory in an efficient way. Our observations show that the same phonetic identity may demonstrate various prosodic properties at different positions. For example, word-final unit is lower in pitch and longer in duration than its word-initial counterpart. Also a multi-syllable word may have influence different from a monosyllable word. All these factors have been taken into account in our unit definition.

We defined 5-class units as follows.

- Class 1: regular consonant + vowel, semi-vowel + vowel, nasal + vowel. Regular consonant includes stop, fricative and dental etc.

- Class 2: Class 1 + nasal/semi-vowel

- Class 3: sub-CV It is a part of Class 1 or Class 2.

- Class 4: consonant initial and consonant initial group. Consonant initial is a consonant acting as syllable initial while consonant initial group mainly refers to a stop followed by a dental.(for example, $s+t$ )

- Class 5: consonant final and consonant final group

\section{UNIT SELECTION}

As mentioned in [4], cost function reflects cost in unit substitution and transition. Here we deal with two categories of costs: unit cost and transition cost. The unit cost includes left unit cost and right unit cost, while the transition cost contains intra-syllable cost and inter-syllable cost. These cost functions guide the search procedure to select the optimal sequence of candidate phoneme for a target word.

\subsection{Unit cost}

We classify all the candidate phonemes into 4 classes, namely vowel, fricative, stop and nasal, based on the place of articulation and manner of articulation. Each class has left and right unit cost, which are empirical values. If the left neighbor of a candidate phoneme has the same phonetic class as the left neighbor of the target phoneme, the left unit cost of the candidate is set to zero. So is the right unit cost. We have the phonetic classification and cost value nearly similar to [4]. 
In the previous work[4], researchers focus on phonetic classification but do not take prosodic position into account explicitly. In our synthesis approach, all the carrier sub-words come from the pre-recorded continuous speech. We observed the same phonetic identity demonstrates various prosodic features. To reflect these differences, we define syllable position and phoneme position as follows.

\section{Syllable position}

$$
\begin{array}{ll}
\text { - } & W I, \text { syllable is at word initial } \\
\text { - } & W M, \text { syllable is at word medial } \\
\text { - } & W F, \text { syllable is at word final } \\
\text { - } & W S, \text { syllable is within a monosyllable word }
\end{array}
$$

\section{Phoneme position}

$$
\begin{array}{ll}
\text { - } & S I, \text { phoneme is at syllable initial } \\
\text { - } & S M \text {, phoneme is at syllable medial } \\
\text { - } & S F \text {, phoneme is at syllable final } \\
\text { - } & S S \text {, phoneme is a separate syllable }
\end{array}
$$

\section{Position sharing}

We adopt cost matrix to describe the potential risk for position substitution. The bigger the prosodic difference is, the higher the cost value is. The table-1 describes cost values for syllable position sharing.

Table-1 The cost matrix for syllable position sharing

\begin{tabular}{|l|l|l|l|l|}
\hline & WI & WM & WF & WS \\
\hline WI & 0 & 4000 & 20000 & 20000 \\
\hline WM & 4000 & 0 & 20000 & 25000 \\
\hline WF & 20000 & 20000 & 0 & 4000 \\
\hline WS & 20000 & 25000 & 4000 & 0 \\
\hline
\end{tabular}

As for phoneme position sharing, we notice that unit cost can reflect the difference of phonetic context but it does not cover the influence of syllable boundary change. Specifically, $p$ in crippled and $p$ in crippling can be an instance. The table-2 describes the corresponding cost matrix.

Table-2 The cost matrix for phoneme position sharing

\begin{tabular}{|l|l|l|l|l|}
\hline & SI & SM & SF & SS \\
\hline SI & 0 & 2000 & 4000 & 4000 \\
\hline SM & 2000 & 0 & 4000 & 4000 \\
\hline SF & 4000 & 4000 & 0 & 2000 \\
\hline SS & 4000 & 4000 & 2000 & 0 \\
\hline
\end{tabular}

\subsection{Transition cost}

Transition cost reflects cost in phoneme transition. If two contiguous candidate phonemes occur in the same carrier subword, the corresponding transition cost is zero. We consider 2 sorts of transition cost: inter-syllable cost and intra-syllable cost. In our practice, intra-syllable cost plays a greater role than inter-syllable cost for in-word prosody. We adopt 5 phonetic classes and 6 phonetic classes as illustrated in Table 5 for intra-syllable cost and inter-syllable cost, respectively.
In short, for any candidate phoneme $p_{i}$, its cost $c_{i}$ satisfies

$$
c_{i}=u l_{i}+u r_{i}+\operatorname{tran}_{i}+w_{i}+s_{i}(1)
$$

where

$u l_{i}$ : left unit cost; $u r_{i}$ : right unit cost;

tran $_{i}$ : concatenation cost.

$w_{i}$ : cost for syllable position;

$s_{i}$ : cost for phoneme position.

\subsection{Search procedure}

For each target phoneme, there are one or more candidate phonemes in the inventory. We can compute the cost value according to (1). Thus, we can construct a trellis for a target word. For instance, Fig. 1. represents a trellis corresponding to the word, synthesis. The issue of unit selection can be converted into an "optimal" search problem. In[4], the search procedure is based on Viterbi decoding. Strictly speaking, Viterbi decoding is an asymptotically optimal solution rather than a true optimal search algorithm. The search result is always dominated by the beginning portion of a path(we call it prefix preference). To resolve this problem, we develop a search procedure based on dynamic programming.

Target word: synthesis Pronunciation: s'ihnthaxsaxs

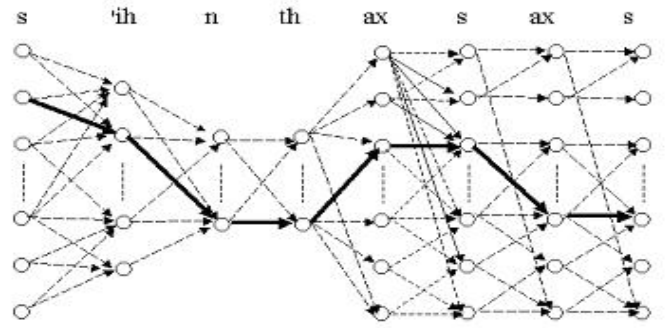

Figure 1 Illustration of the search procedure for syhtesis 1

\subsubsection{Induction process for unit selection}

We view each phoneme of the target word as a time instant and add one virtual node, the start node $q_{s}$ corresponding to time 0 before time 1 (corresponding to the first phoneme). All the transitions from $q_{s}$ to nodes in the first stage (time 1) have cost of zero. We denote the optimal path from $q_{s}$ to the node $q_{i}$ of time $t$ as path $(t, i)$. Consequently, path $(t, i)$ is one set of sequential states. Accordingly, we denote the cost summation of path $(t, i)$ as $s(t, i)$. Suppose the target word has $T$ phonemes and the $t$-th phoneme has $N_{t}$ candidates, we can define the induction process as follows.

initialization:

$$
\begin{aligned}
& s(0,0)=0, \\
& \operatorname{path}(0,0)=\left\{q_{s}\right\} .
\end{aligned}
$$


induction:

$$
\begin{aligned}
& \text { given } j, \quad s(t, j)=\min _{1 \leq i \leq N_{t}}\left[s(t-1, i)+c_{j}\right], 1 \leq \mathrm{t} \leq \mathrm{T}, \\
& \text { denotes } k=\arg \min _{1 \leq i \leq \mathrm{N}_{\mathrm{t}}}\left[s(t-1, i)+c_{j}\right], \text { then } \\
& \text { path }(t, j)=\operatorname{path}(t-1, k) \cup\{k\} .
\end{aligned}
$$

termination:

$$
\begin{aligned}
& \text { at time } T, \quad k=\arg \min _{1 \leq i \leq N_{T}} s(T, i) . \\
& \text { then path }(T, k)-\left\{q_{s}\right\} \text { is the optimal path. }
\end{aligned}
$$

\subsubsection{Complexity analysis}

It is well understood that the time complexity of dynamic programming is concerned with edge/arc number. In the induction phase, when transiting from time $t$ to time $t+1$, our approach requires calculations among which each state needs $2 N_{t} \cdot N_{t+1}$ calculations, so the overall time complexity is $O(N)$, where $N=\sum_{t=1}^{T-1} N_{t} \cdot N_{t+1}$. In the same way, the space complexity is also $O(N)$.

\section{SPEECH SYNTHESIS}

Although concatenative speech synthesis has been proved to be a promising approach to practical TTS systems, the waveform discontinuity at concatenating points or between two contiguous units can result in audible artifact. The speech smoothing at concatenating points is a key to achieving highly natural and intelligible speech synthesis. Here we propose a method to realize the smooth transition at concatenating points effectively. We develop a method to determine concatenating points between the contiguous units based on a cost measure. The units for concatenation are represented by two components: vocal tract response and excitation. With the two components, the major speech production features vocal tract response, pitch value and phase are interpolated and merged at concatenating point respectively. By filtering the merged excitation through the modified synthesis filter, the smooth synthetic transition between two units can be generated. A detailed diagram is shown in Fig. 3.

\subsection{Determining an accurate concatenating point}

Many kinds of objective measure have been explored for the automatic detection of the audible discontinuities at the unit concatenation point. Perceptual test can be employed to evaluate their detection rate of concatenation discontinuity by manual listening. Motivated by speech recognition methods, many approaches to unit selection in speech synthesis employ the Euclidean distance between Mel-Frequency Cepstral Coefficient (MFCC). Perceptual test shows that this distance is an effective objective measure of audible discontinuity.

A concatenation cost function (CCF) based on Euclidean distance measure of MFCC is defined to estimate the concatenating cost between two frames ( $\mathrm{X}$ and $\mathrm{Y}$ ) at the signal level. By minimizing the CCF measure between the concatenating units, a relatively accurate concatenating position can be allocated.

In order to reduce the computational complexity of the $\mathrm{CCF}$ calculation in the run time, the MFCC coefficients may be obtained for the end frame and $N$ head frames respectively for each unit, as shown in Fig. 2.

During concatenating synthesis, the CCFs (between the end frame $T$ of the previous unit and head frames $H_{i}$ of the successive unit, $0 \leq i \leq N)$ are calculated. The frame $u$ of the successive unit with the lowest CCF value is selected to concatenate or merge with the previous unit.

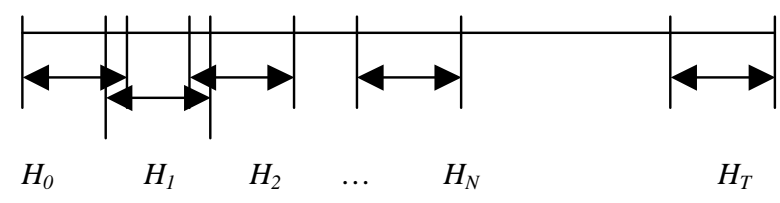

Figure 2 Calculating the MFCC for several frames at head and end of unit

\subsection{Compressive coding of the sound inventory}

All acoustic units of TTS sound inventory are encoded with a high-quality vocoder. It significantly reduces the memory requirement of the final TTS inventory and builds up a compact speech database for TTS.

\subsection{Decoding the parametric bit-stream of the units for concatenation}

When concatenating the units, the parametric bit-stream of units for concatenation are extracted and decoded into two major components: vocal tract parameters LSP (Line Spectral Pairs - a kind of representation of vocal tract response) $L S P[i]$ for frame $i$ and excitation stream Exc[i]. The pitch estimation $P i$ of each frame $i$ is also obtained.

\subsection{Interpolating or merging the major speech production features}

The pitch values of two units at boundary are linearly interpolated to perform smoothing. With the smoothed pitch values of the previous unit and the succeed unit, we can shift concatenating point by deleting several samples at the head frame of the successive unit to keep the base harmonic phase continuously. With the newly refined concatenating point at the head of the successive unit, its first pitch cycle excitation signal is merged into a new cycle with the last pitch cycle of the previous unit.

The LSP vector corresponding to the merged pitch cycle excitation segment is obtained by interpolating the last LSP vector of the previous unit and the first LSP vector of the successive unit. The concatenated LSP vector series is built up then. 1 


\subsection{Reconstructing the smooth synthetic speech signal}

The concatenated LSP vector series of the contiguous units are converted to predictive coefficients. The smoothed synthetic speech is reconstructed by feeding the concatenated smooth excitation series to the synthesis filter that is constructed with the predictive coefficient.

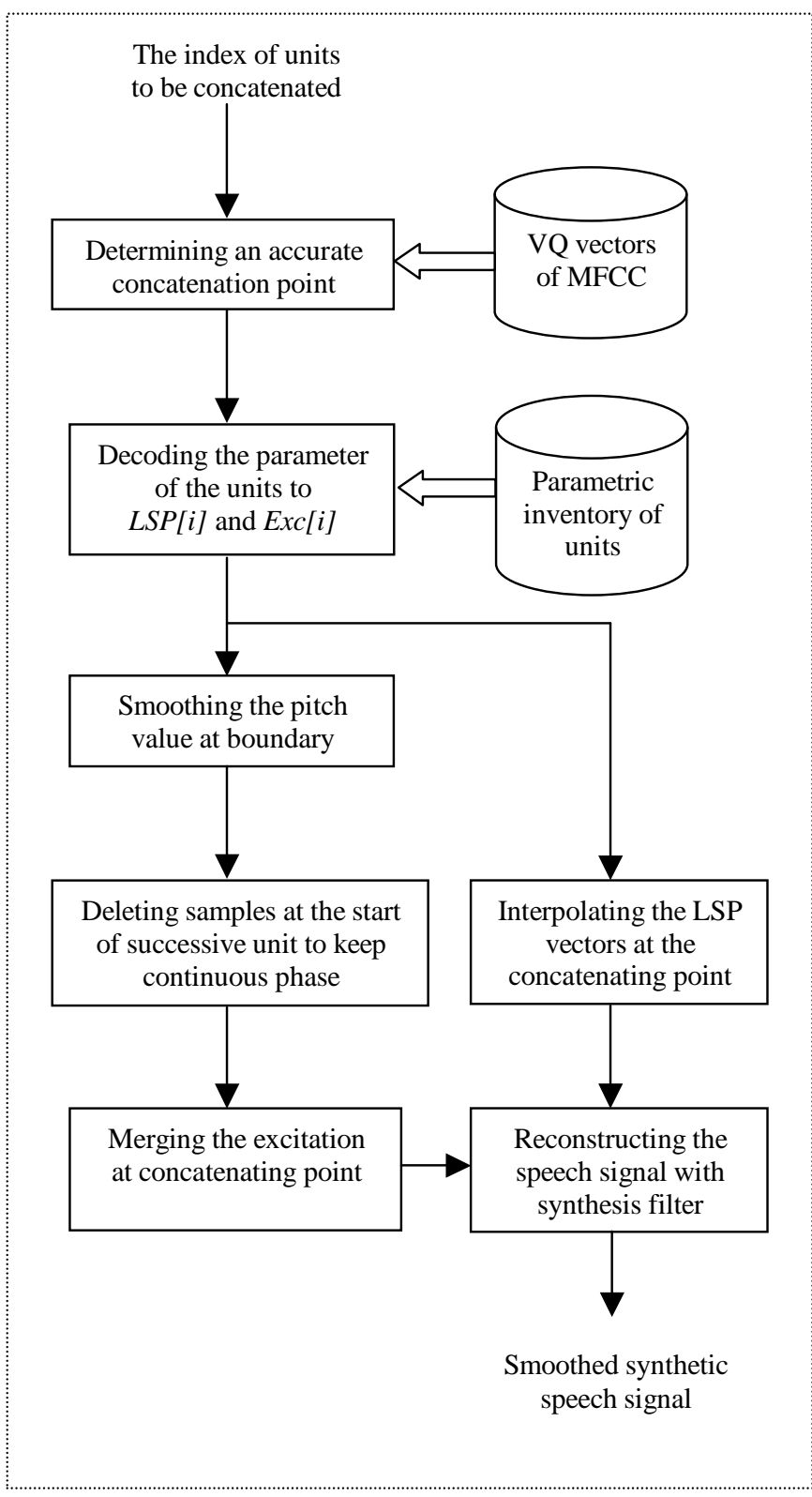

Figure 3 Speech smoothing for the concatenative speech synthesis

\section{TEST}

To build up a complete embedded TTS engine for evaluation, we implemented a TTS front end and the speech synthesizer. The front end consists of several modules, including text normalization, letter-to-sound conversion, automatic phrasing and the mentioned unit selection. We conducted a preliminary listening test. To investigate the concatenation effect, we selected 1,080 words from sentence medial and 1,187 words from sentence final in a corpus. These selected words have high phonetic balance and cover all of our target concatenation points. The experimental results show that our TTS engine can achieve satisfactory naturalness. The engine size is less than $900 \mathrm{~KB}$. We chose a formant-based TTS engine to perform a comparison. It occupies comparable memory space and runs under the same platform. We used 30 articles from several domains as test material. Our TTS engine outperforms the formant-based one noticeably, 95\% of subjects preferred our engine in both naturalness and intelligibility.

\section{DISCUSSION}

Concatenative synthesis can generate high-quality synthetic speech, which can overcome some problems existing in formant-based synthesis, such as mechanical sound. However, it often requires large memory space and somewhat lacks flexibility in modification. In our work, the well-designed speech inventory can provide prosodic information implicitly and lower the need of speech modification in the synthesis stage. Speech smoothing can remove or weaken the discontinuity at concatenating points. We are improving the text processing part under the footprint constraint to overcome the fragmental prosody in some cases that are caused by improper phrasing.

\section{ACKNOWLEDGE}

We are grateful to Daisy Chen for her effective work in data preparation. In addition, more than ten interns evaluated our engines and gave us constructive feedback. Also Ke-Song Han contributed to our text-normalization module substantially. In particular, we owe thanks to Jian-Cheng Huang for his insightful comments and suggestions.

\section{REFERENCES}

[1] Black, Alan W., Lenzo, Kevin A., Flite: A Small Fast Run-time Synthesis Engine, In SSW4-2001, paper 204. [2] Black, A., and Lenzo, K. Building Voices In the Festival Speech Synthesis System, http://festvox.org, 2000.

[3] Hertz, S. R., Integration of Rule-based Formant Synthesis and Waveform Concatenation: A Hybrid Approach to Textto-Speech Synthesis, Proceedings of 2002 IEEE Workshop on Speech Synthesis, 11-13 Sept. 2002, Pages:87-90

[4] Jon Rong-Wei Yi, Natural-Sounding Speech Synthesis Using Variable-Length Units, Master Thesis, MIT, 1997

[5] Stevens, K. N., Toward Formant Synthesis With Articulatory Controls, Proceedings of 2002 IEEE Workshop on Speech Synthesis, 11-13 Sept. 2002, Pages:67-72 